\title{
Correlation of Vitamin D Serum Level of Mothers and Their Newborns
}

\author{
Hassan Boskabadi (iD ${ }^{1}$ and Elahe Heidari (iD) ${ }^{1, *}$ \\ ${ }^{1}$ Department of Pediatrics, Faculty of Medicine, Mashhad University of Medical Sciences, Mashhad, Iran \\ "Corresponding author: Department of Pediatrics, Faculty of Medicine, Ghaem Hospital,Mashhad University of Medical Sciences, Mashhad, Iran. Tel: +98-9155574738, Email: \\ heidarie@mums.ac.ir \\ Received 2021 April 25; Revised 2021 July 06; Accepted 2021 July 14.
}

\begin{abstract}
Background: The possibility of vitamin D deficiency is high among mothers and their premature newborns.

Objectives: Due to the high rate of vitamin D deficiency in Iranian women, this study aimed to determine the association between the serum levels of vitamin D in the blood of premature newborns and their mothers in the Iranian population.

Methods: This cross-sectional study was conducted on 324 mothers and their 324 newborns with gestational age less than 37 weeks, who were referred to Ghaem Hospital affiliated to Mashhad University of Medical Sciences, Mashhad, Iran, during 2016-2020. After clamping, 1.5 cc blood samples were obtained through the umbilical cord to determine the neonates' vitamin D levels. Finally, the serum levels of vitamin D were measured, and the correlation between the levels of vitamin D of mothers and their newborns was calculated.

Results: The mean vitamin D levels of newborns and mothers were $15.75 \pm 11.18$ and $20.16 \pm 13.41 \mathrm{ng} / \mathrm{mL}$, respectively. Moreover, there was a high correlation between the vitamin D levels of mothers and their newborns $(r=0.672 ; P<0.001)$. Furthermore, a significant correlation was observed between the vitamin D levels of mothers, who used vitamin $\mathrm{D}$ supplementation during pregnancy and their neonates' levels $(\mathrm{r}=0.773 ; \mathrm{P}<0.001)$.

Conclusions: The findings suggest a positive correlation between neonatal and maternal vitamin D levels. Moreover, it seems that maternal vitamin D levels can predict neonatal vitamin D deficiency.
\end{abstract}

Keywords: Vitamin D, Neonate, Supplements, Maternal, Prematurity

\section{Background}

Prematurity is associated with increased neonatal and childhood morbidity and mortality (1). Premature neonates weighing less than $1,500 \mathrm{~g}$ have far more problems and need more intensive care. According to the studies performed in Iran, about $28 \%$ of neonatal mortality is due to prematurity $(2,3)$. The probability of the survival rate of high-risk infants, including premature infants, has increased recently with the advancement of perinatology (4).

Vitamin D deficiency or hypovitaminosis D is a major public health problem, especially among pregnant women. The prevalence of vitamin D deficiency varies in different regions worldwide relative to the place of residence and local clothing style, ranging between $18 \%$ and $84 \%(5,6)$. The possibility of vitamin D deficiency is high among premature newborns $(7,8)$.

Pregnancy is a risk factor for vitamin D deficiency; accordingly, it can lead to an extra load reducing 25hydroxyvitamin D levels in pregnant women (9). Serum 25-hydroxyvitamin D levels less than $25 \mathrm{ng} / \mathrm{mL}$ during preg- nancy are estimated at $17 \%-18 \%, 12 \%-34 \%$, and $80 \%$ in Caucasian (10), Indian (10), and Iranian women (11), respectively. Moreover, the level for pregnant mothers in China was found to be $28 \%$ - 54\% nmol/L (12-14). Decreased levels of mothers' vitamin D can reduce the transmission of vitamin D through the placenta. Therefore, the newborns' storage of vitamin D decreases at birth (15).

Vitamin D is a steroid hormone that has been known for years to play a classic role in mineral balance, bone metabolism, and neuromuscular function. Moreover, vitamin $\mathrm{D}$ has a remarkable effect on the regulation of more than 1,000 genes involved in biological processes, angiogenesis, inflammation, and immune function $(16,17)$. The concentration of vitamin $\mathrm{D}$ in the fetus depends on the concentration of vitamin D in the mother. Vitamin D deficiency is common during pregnancy and is associated with an increased risk of fetal growth restriction, sepsis, and lower respiratory infections (18-20). It is worth mentioning that this vitamin has a significant influence on many neonatal diseases, including neonatal respiratory distress syndrome, cerebral hemorrhage, and premature retinopa- 
thy $(21,22)$.

The serum vitamin D measurement in pregnancy is one of the factors to predict vitamin D deficiency (23), which leads to poor bone development. Therefore, vitamin D intake is included in the current daily recommendations to reduce the prevalence and prevent additional consequences of low serum vitamin D levels $(7,8)$. Knowing about vitamin D physiologic components, risk factors of vitamin D deficiency and methods of supplementation to attain optimal levels in pregnant and lactating women would help to prevent and reduce illnesses linked to early vitamin D deficiency (15).

\section{Objectives}

As mentioned above, mothers' vitamin D deficiency during pregnancy can be detrimental to the health of newborns. In this regard, it is essential to investigate the factors associated with newborns' vitamin D deficiency. Due to the high rate of vitamin D deficiency in Iranian women, this study aimed to determine the association between serum levels of vitamin D in the blood of premature newborns and their mothers in the Iranian population. Moreover, this study attempted to investigate the relationship between vitamin D supplement consumption during pregnancy and the serum levels of vitamin D in premature newborns.

\section{Methods}

This single-center, cross-sectional study was conducted on premature newborns who were referred to Ghaem Hospital affiliated to Mashhad University of Medical Sciences, Mashhad, Iran, during 2016 - 2020.

\subsection{Inclusion and Exclusion Criteria}

The inclusion criteria were mothers and their newborns with gestational age less than 37 weeks. On the other hand, epileptic and asthmatic mothers, as well as their newborns, were excluded from the study.

\subsection{Study Design}

The study population included 324 mothers and their 324 premature newborns with gestational age less than 37 weeks, who were born in Ghaem Hospital, Mashhad, Iran. According to the results of the study by Boskabadi et al. (17), the mean \pm SD of vitamin D was reported to be $21.23 \pm$ $15.19 \mathrm{ng} / \mathrm{dL}$ in mothers of the control group and $17.9 \pm 12.55$ $\mathrm{ng} / \mathrm{dL}$ in their infants. Considering alpha of 0.03 and beta of 0.2 , the sample size was estimated to be 317 newborns.
Demographic characteristics, including gestational age, birth weight, first and fifth minute Apgar score, and maternal information, were recorded in questionnaires. After clamping, 1.5 cc blood samples were obtained through the umbilical cord to determine newborns' vitamin D levels. Moreover, 1.5 cc blood samples were obtained from the mothers to assess their vitamin D levels. The prepared samples were centrifuged, and the sera were kept at $-20^{\circ} \mathrm{C}$. Vitamin D level was measured using an Enzyme-Linked Immunosorbent Assay (ELISA) Reader device (RT2100c model, made in Germany) and ELISA washing device. Serum levels of vitamin D below $30 \mathrm{ng} / \mathrm{mL}$ were considered as vitamin D deficiency. To describe the severity of vitamin D deficiency, we used the terms mild, moderate, and severe deficiency for vitamin D serum levels of 20 - 30, 10 - 20, and below $10 \mathrm{ng} / \mathrm{dL}$, respectively (24). Finally, serum levels of vitamin $\mathrm{D}$ and the correlation between the levels of vitamin $\mathrm{D}$ in mothers and their newborns were measured.

\subsection{Statistical Analysis}

The data were analyzed in SPSS software (version 23). The Pearson correlation was used to determine the relationship between variables. A P-value of less than 0.05 was considered statistically significant.

\subsection{Ethical Considerations}

The study protocol was approved by the Ethics Committee of Mashhad University of Medical Sciences, Mashhad, Iran (no.: HYPERLINK "http://research.mums.ac.ir/main/cartable.action"960406) IR.MUMS.fm.REC.1396.635. Consent was obtained from neonates' parents before doing the research. In line with the principles of research ethics, a code was given to ensure the confidentiality of data.

\section{Results}

In this study, we assessed 324 serum levels of vitamin D in mothers and their neonates. There was a significant correlation between the vitamin $D$ levels of mothers and their newborns $(\mathrm{r}=0.672 ; \mathrm{P}<0.001)$. Regarding the descriptive statistics, the majority of the newborns $(52.5 \%, \mathrm{n}=168)$ were males. Moreover, the mean age of the mothers was $29.6 \pm$ 6.64 years. Table 1 indicates the demographic characteristics, including maternal age, gestational age, and neonatal birth weight. The assessment of the type of delivery showed that 79 (30.9\%) mothers had normal vaginal deliveries, and 177 (69.1\%) mothers had cesarean sections. Moreover, the mean vitamin D levels of newborns and mothers 


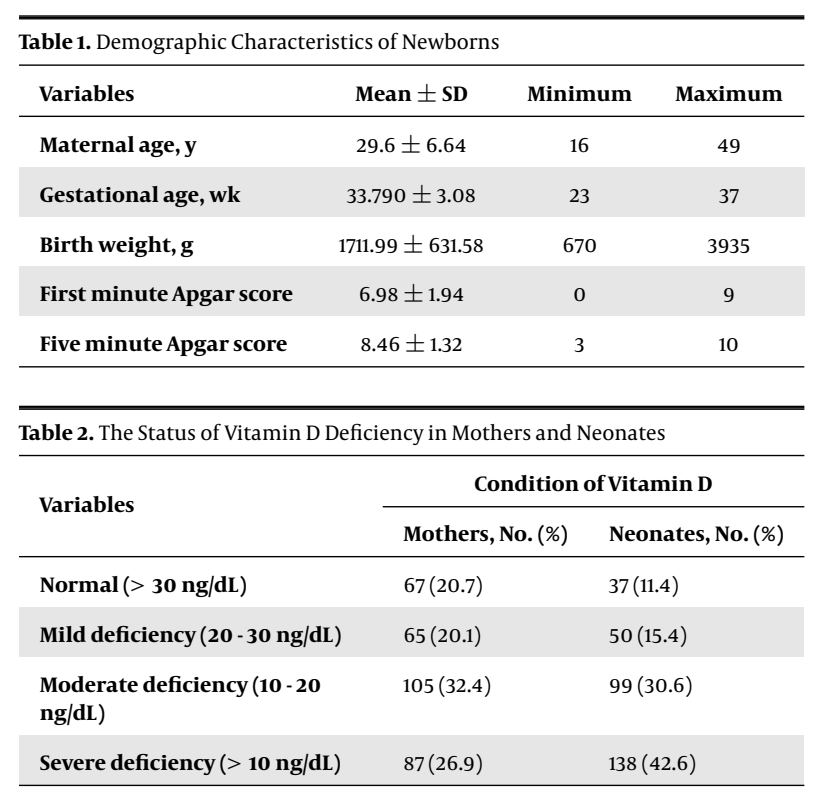

were $15.75 \pm 11.18$ and $20.16 \pm 13.41 \mathrm{ng} / \mathrm{mL}$, respectively (Table 2).

Based on the results, $79.3 \%$ of the mothers and $88.6 \%$ of the newborns had vitamin D deficiency. Table 2 illustrates the status of vitamin D deficiency in mothers and neonates. The analysis of the relationship between vitamin D levels of mothers and their premature newborns revealed a high correlation $(r=0.672 ; \mathrm{P}<0.001)$ (Figure 1$)$. A high correlation was also observed between the vitamin $D$ levels of mothers who did not take vitamin D supplementation during pregnancy and those of their newborns ( $\mathrm{r}=$ 0.61; P $<0.001$ ) (Figure 1).

\section{Discussion}

To reiterate, $80 \%$ of the mothers and $89 \%$ of their newborns had vitamin D deficiency. The prevalence of vitamin D deficiency in preterm neonates is high in the Middle East compared to western countries (25). According to a study conducted by Merewood et al. (26), vitamin D deficiency was present in $58 \%$ of newborns and $35.8 \%$ of mothers. Moreover, $38 \%$ of newborns and $23.1 \%$ of mothers suffered from severe vitamin D deficiency. The frequency of vitamin D deficiency in the present study was higher than that in the aforementioned study. Merewood et al. (26) also found a close association of vitamin D deficiency with winter birth and BMI. Vitamin D deficiency was identified in nearly $100 \%$ of mothers who had newborns with rickets, compared to just over $50 \%$ of mothers who gave birth to newborns without rickets in another study conducted in the Middle East (27). This difference is due to the lack of vitamin D stores at birth in newborns whose mothers were vitamin D deficient. It should be mentioned that the breast milk of these mothers contains negligible amounts of vitamin D (28).

Moreover, about a quarter of pregnant women with preterm birth were severely deficient in vitamin D. It was also observed that 43 (13\%) newborns had severe vitamin D deficiency. According to a study carried out by Dawodu and Nath (25), a relationship was found between the high prevalence of maternal vitamin D deficiency and vitamin D stores of prematurely born Arab neonates. The obtained result was confirmed in the community (29). In another study, serum 25-hydroxyvitamin D concentrations of less than $12.5 \mathrm{nmol} / \mathrm{L}$ were reported among $44 \%$ of preterm infants, which is compatible with the metabolic bone disease (30).

Based on the obtained results of the present study, there was a high correlation between the vitamin D levels of premature newborns and those of their mothers. Moreover, a significant correlation was observed between the vitamin D levels of mothers who used vitamin D supplementation during pregnancy and those of their premature newborns; also, a high correlation was reported between non-supplemented mothers and their premature newborns in terms of vitamin D level. In a study conducted by Wang et al., neonatal serum 25-hydroxyvitamin D levels were significantly correlated with maternal serum 25hydroxyvitamin D levels. They showed that the maternal serum 25-hydroxyvitamin D levels can help predict neonatal vitamin $\mathrm{D}$ deficiency. The area under the curve was 0.96 , and both sensitivity (97.2\%) and specificity of maternal serum 25-hydroxyvitamin D levels were high to predict neonatal vitamin D deficiency (80.3\%) (31). The results of a study conducted by Zhu et al. (12) showed an association between the maternal vitamin $\mathrm{D}$ level and the incidence of preterm delivery. Accordingly, lower vitamin D was reported among mothers who delivered their babies before 31 weeks compared to those delivered after 31 weeks. In the mentioned study, vitamin D deficiency was observed in $63 \%$ of mothers who had preterm babies (12). There are two main sources of vitamin $\mathrm{D}$, namely exposure to solar radiation and dietary intake (32). As we know, the prevalence of vitamin D deficiency depends on the place of residence and local clothing style $(5,6)$.

Since insufficient maternal vitamin D levels may lead to neonatal vitamin D deficiency, which is higher among preterm birth, it is of utmost importance to use vitamin D supplementation during pregnancy as a strategy to maintain vitamin D sufficiency in both mothers and their newborns (33). The American Academy of Pediatrics introduced guidelines for vitamin $\mathrm{D}$ intake to prevent rickets and vitamin $\mathrm{D}$ deficiency in healthy neonates. In this guideline, a daily supplement of 400 IU/day of vitamin D 


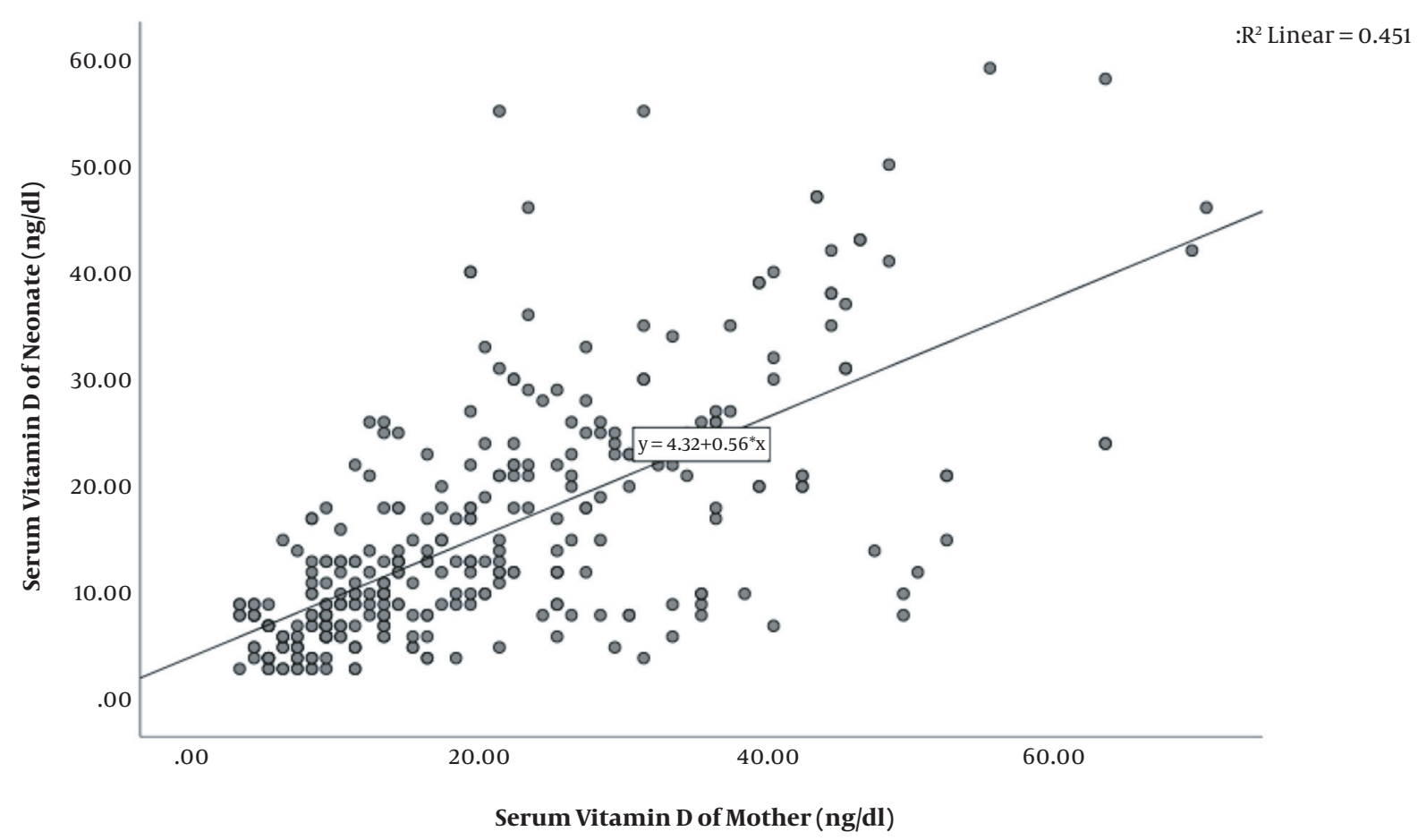

Figure 1. Correlation between mothers and their newborns in terms of vitamin D levels

should be given to newborns from the first few days of life and continued throughout childhood (34).

Based on our findings, there was a high correlation between the vitamin D levels of supplemented mothers and those of their newborns, and a significant correlation was observed between non-supplemented mothers and their newborns in terms of vitamin D levels. According to the literature, there is a difference between supplemented and non-supplemented mothers regarding serum 25-hydroxyvitamin D levels (35), and the use of vitamin D is suggested for preterm newborns. The recommended rate of vitamin D supplementation varies and ranges from 150 - 400 to 800 - $1600 \mathrm{IU} /$ day (25). Moreover, up to $400 \mathrm{IU} /$ day is recommended to maintain normal vitamin D levels in preterm infants (36).

According to our findings, both mothers who did not take vitamin D supplementation during pregnancy and who used vitamin D supplementation during pregnancy had vitamin D levels in high correlation with those of their newborns, but this does not mean that taking vitamin D supplement has little effects. This finding can be justified as follows. The consumption of vitamin D in the third trimester of pregnancy in mothers increased its serum level in the mother, but due to the high vitamin D demand of fetal tissues, this vitamin was absorbed by fetal tissues, and the serum levels in the fetus did not increase as much as we expected. Some studies have shown that injecting vitamin D into the mother during delivery, despite not increasing the serum levels of vitamin D in the newborn, has led to the benefits of receiving vitamin $\mathrm{D}$, such as reducing neonatal respiratory distress (37).

To conclude, the optimal vitamin D requirement of preterm newborns should be determined considering mothers' vitamin D levels during pregnancy and geographical locations (25). Due to the high prevalence of vitamin D deficiency among Iranian women, vitamin D supplementation should be used to ensure vitamin D sufficiency in high-risk infants, including prematurely born neonates.

Limitations of the Study: Although this study paved the way for the evaluation of vitamin D levels in premature newborns, it suffers some limitations. This was a singlecenter study; therefore, it is not possible to generalize our data to other populations. Moreover, due to the observational nature of our study, it was not possible to achieve a causal relationship between variables. There is also a possibility of data manipulation by confounding variables. It also would be beneficial to check the seasons when the babies were born to assess the relationship between the season of birth and vitamin D levels. 


\subsection{Conclusions}

Vitamin D deficiency is very common among mothers and their premature newborns. The maternal and neonatal vitamin D levels are positively correlated. It seems that maternal vitamin D levels can predict neonatal vitamin D deficiency. Checking serum levels of vitamin D in women who are planning to be pregnant or at the first visit of pregnancy is recommended. Further research investigating the relationship between the birth season and vitamin D levels of mothers and newborns would be a useful follow-up to this study.

\section{Footnotes}

Authors' Contribution: HB contributed to the conception and design of the research, acquisition, and analysis of the data. EH contributed to the interpretation of the data and manuscript drafting.

Conflict of Interests: The authors declare that they have no competing interests.

Ethical Approval: The Ethics Committee of the Mashhad University of Medical Sciences approved this study (code of ethics: IR.MUMS.fm.REC.1396.635).

Funding/Support: This study was supported by the Mashhad University of Medical Sciences, Mashhad, Iran.

Informed Consent: Informed consent was obtained from all patients in the study.

\section{References}

1. Abrishami M, Maemori GA, Boskabadi H, Yaeghobi Z, Mafi-Nejad S, Abrishami M. Incidence and risk factors of retinopathy of prematurity in mashhad, northeast iran. Iran Red Crescent Med J.2013;15(3):22933. doi: 10.5812/ircmj.4513. [PubMed: 23984003]. [PubMed Central: PMC3745752].

2. Rezaeian A, Boskabadi H, Mazlom SR. Factors associated with perinatal mortality in preterm infants in NICU Ghaem Hospital, Mashhad. I North Khorasan Univ Med Sci. 2012;4(3):349-60. doi: 10.29252/jnkums.4.3.349.

3. Behnam Vashani H, Askari Hoseini Z, Boskabadi H, Rezaeian A. The ef fect of family participation on mother's general health and length of hospitalization of premature neonate. Evid Based Care. 2014;4(1):5360.

4. Chen W, Hu J, Bouwstra S, Oetomo SB, Feijs L. Sensor integration for perinatology research. Int J Sensor Networks. 2011;9(1). doi: 10.1504/ijsnet.2011.037303.

5. Vandevijvere S, Amsalkhir S, Van Oyen H, Moreno-Reyes R. High prevalence of vitamin D deficiency in pregnant women: a national cross-sectional survey. PLoS One. 2012;7(8). e43868. doi 10.1371/journal.pone.0043868. [PubMed: 22937114]. [PubMed Central PMC3427250].

6. Dawodu A, Wagner CL. Mother-child vitamin D deficiency: an international perspective. Arch Dis Child. 2007;92(9):737-40. doi: 10.1136/adc.2007.122689. [PubMed: 17715433]. [PubMed Central: PMC2084036].
7. Wagner CL, Greer FR, American Academy of Pediatrics Section on B, American Academy of Pediatrics Committee on N. Prevention of rickets and vitamin D deficiency in infants, children, and adolescents. Pediatrics. 2008;122(5):1142-52. doi: 10.1542/peds.2008-1862. [PubMed: 18977996].

8. Holick MF. Vitamin D deficiency. N Engl J Med. 2007;357(3):266-81. doi: 10.1056/NEJMra070553. [PubMed: 17634462].

9. Fernandez-Alonso AM, Dionis-Sanchez EC, Chedraui P, GonzalezSalmeron MD, Perez-Lopez FR, Spanish Vitamin D, et al. Firsttrimester maternal serum 25-hydroxyvitamin $\mathrm{D}(3)$ status and pregnancy outcome. Int J Gynaecol Obstet. 2012;116(1):6-9. doi: 10.1016/j.ijgo.2011.07.029. [PubMed: 21959069].

10. Sahu M, Bhatia V, Aggarwal A, Rawat V, Saxena P, Pandey A, et al. Vitamin D deficiency in rural girls and pregnant women despite abundant sunshine in northern India. Clin Endocrinol (Oxf). 2009;70(5):680-4. doi: 10.1111/j.1365-2265.2008.03360.x. [PubMed: 18673464].

11. Bassir M, Laborie S, Lapillonne A, Claris O, Chappuis MC, Salle BL. Vitamin D deficiency in Iranian mothers and their neonates: a pilot study. Acta Paediatr. 2001;90(5):577-9. [PubMed: 11430721].

12. Zhu T, Liu TJ, Ge X, Kong J, Zhang LJ, Zhao Q. High prevalence of maternal vitamin $\mathrm{D}$ deficiency in preterm births in northeast China, Shenyang. Int J Clin Exp Pathol. 2015;8(2):1459-65. [PubMed: 25973031]. [PubMed Central: PMC4396334].

13. Song SJ, Si S, Liu J, Chen X, Zhou L, Jia G, et al. Vitamin D status in Chinese pregnant women and their newborns in Beijing and their relationships to birth size. Public Health Nutr. 2013;16(4):687-92. doi: 10.1017/S1368980012003084. [PubMed: 23174124].

14. Wang J, Yang F, Mao M, Liu DH, Yang HM, Yang SF. High prevalence of vitamin D and calcium deficiency among pregnant women and their newborns in Chengdu, China. World J Pediatr. 2010;6(3):265-7. doi: 10.1007/s12519-010-0224-x. [PubMed: 20706824].

15. Onwuneme C, Martin F, McCarthy R, Carroll A, Segurado R, Murphy J, et al. The Association of Vitamin D Status with Acute Respiratory Morbidity in Preterm Infants. J Pediatr. 2015;166(5):1175-1180 e1. doi: 10.1016/j.jpeds.2015.01.055. [PubMed: 25919726].

16. Umar M, Sastry KS, Chouchane AI. Role of Vitamin D Beyond the Skeletal Function: A Review of the Molecular and Clinical Studies. Int J Mol Sci. 2018;19(6). doi: 10.3390/ijms19061618. [PubMed: 29849001]. [PubMed Central: PMC6032242].

17. Boskabadi H, Mamoori G, Khatami SF, Faramarzi R. Serum level of vitamin D in preterm infants and its association with premature-related respiratory complications: a case-control study. Electron Physician. 2018;10(1):6208-14. doi: 10.19082/6208. [PubMed: 29588821]. [PubMed Central: PMC5853995].

18. Burris HH, Rifas-Shiman SL, Camargo CJ, Litonjua AA, Huh SY, Rich-Edwards JW, et al. Plasma 25-hydroxyvitamin D during pregnancy and small-for-gestational age in black and white infants. Ann Epidemiol. 2012;22(8):581-6. doi:10.1016/j.annepidem.2012.04.015. [PubMed: 22658824]. [PubMed Central: PMC3396717].

19. Wayse V, Yousafzai A, Mogale K, Filteau S. Association of subclinical vitamin $\mathrm{D}$ deficiency with severe acute lower respiratory infection in Indian children under 5 y. Eur J Clin Nutr. 2004;58(4):563-7. doi: 10.1038/sj.ejcn.1601845. [PubMed: 15042122].

20. Yang LR, Li H, Yang TY, Zhang T, Zhao RC. [Relationship between vitamin D deficiency and early-onset neonatal sepsis]. Zhongguo Dang Dai Er Ke Za Zhi. 2016;18(9):791-5. [PubMed: 27655531]. [PubMed Central: PMC7389977].

21. Boskabadi H, Zakerihamidi M, Faramarzi R. The vitamin D level in umbilical cord blood in premature infants with or without intra-ventricular hemorrhage: A cross-sectional study. Int J Reprod Biomed. 2018;16(7):429-34. [PubMed: 30234182]. [PubMed Central: PMC6129378].

22. Boskabadi H, Maamouri G, Hemmatipour A, Parvini Z, Ramazani A, Bagheri F. Comparison of Serum Vitamin D in the Umbilical Cord of Survived with Not Survived Premature Infants. Iran J Pediatr. 2019;29(3). doi: 10.5812/ijp.84798. 
23. Bodnar LM, Simhan HN, Powers RW, Frank MP, Cooperstein E, Roberts JM. High prevalence of vitamin D insufficiency in black and white pregnant women residing in the northern United States and their neonates. J Nutr. 2007;137(2):447-52. doi: 10.1093/jn/137.2.447. [PubMed: 17237325]. [PubMed Central: PMC4288960].

24. Boskabadi H, Maamouri G, Kalani-Moghaddam F, Ataee Nakhaei MH, Zakerihamidi M, Rakhshanizadeh F. Comparison of Umbilical Cord Serum Vitamin D Levels between Infants with Transient Tachypnea of the Newborn and those without Respiratory Distress. Arch Iran Med. 2020;23(8):530-5. doi: 10.34172/aim.2020.55. [PubMed: 32894964].

25. Dawodu A, Nath R. High prevalence of moderately severe vitamin D deficiency in preterm infants. Pediatr Int. 2011;53(2):207-10. doi: 10.1111/j.1442-200X.2010.03209.x. [PubMed: 20667028].

26. Merewood A, Mehta SD, Grossman X, Chen TC, Mathieu JS, Holick $\mathrm{MF}$, et al. Widespread vitamin D deficiency in urban Massachusetts newborns and their mothers. Pediatrics. 2010;125(4):640-7. doi: 10.1542/peds.2009-2158. [PubMed: 20308219].

27. Dawodu A, Agarwal M, Sankarankutty M, Hardy D, Kochiyil J, Badrinath P. Higher prevalence of vitamin D deficiency in mothers of rachitic than nonrachitic children. J Pediatr. 2005;147(1):109-11. doi: 10.1016/j.jpeds.2005.03.001. [PubMed:16027707].

28. Thandrayen K, Pettifor JM. The roles of vitamin D and dietary calcium in nutritional rickets. Bone Rep. 2018;8:81-9. doi: 10.1016/j.bonr.2018.01.005. [PubMed: 29955625]. [PubMed Central: PMC6019962].

29. Amirlak I, Ezimokhai M, Dawodu A, Dawson KP, Kochiyil J, Thomas L, et al. Current maternal-infant micronutrient status and the effects on birth weight in the United Arab Emirates. East Mediterr Health J. 2009;15(6):1399-406. [PubMed: 20218130].

30. Seino Y, Ishii T, Shimotsuji T, Ishida M, Yabuuchi H. Plasma active vitamin D concentration in low birthweight infants with rickets and its response to vitamin D treatment. Arch Dis Child. 1981;56(8):62832. doi: 10.1136/adc.56.8.628. [PubMed: 6973954]. [PubMed Central: PMC1627260].

31. Wang C, Gao JS, Yu SL, Qiu L, Zeng L, Wang DH. [Correlation between neonatal vitamin D level and maternal vitamin D level]. Zhongguo Dang Dai Er Ke Za Zhi. 2016;18(1):20-3. [PubMed: 26781407]. [PubMed Central: PMC7390100].

32. Chen TC, Chimeh F, Lu Z, Mathieu J, Person KS, Zhang A, et al Factors that influence the cutaneous synthesis and dietary sources of vitamin D. Arch Biochem Biophys. 2007;460(2):213-7. doi: 10.1016/j.abb.2006.12.017. [PubMed: 17254541]. [PubMed Central: PMC2698590].

33. Bodnar LM, Rouse DI, Momirova V, Peaceman AM, Sciscione A Spong CY, et al. Maternal 25-hydroxyvitamin d and preterm birth in twin gestations. Obstet Gynecol. 2013;122(1):91-8. doi 10.1097/AOG.ob013e3182941d9a. [PubMed: 23743453]. [PubMed Central: PMC3706065].

34. Kaushal M, Magon N. Vitamin D in pregnancy: A metabolic outlook. Indian J Endocrinol Metab. 2013;17(1):76-82. doi: 10.4103/22308210.107862. [PubMed: 23776856]. [PubMed Central: PMC3659910].

35. Hollis BW, Wagner CL. Assessment of dietary vitamin D requirements during pregnancy and lactation. Am JClin Nutr. 2004;79(5):717-26. doi: 10.1093/ajcn/79.5.717. [PubMed: 15113709].

36. Taylor SN, Wagner CL, Fanning D, Quinones L, Hollis BW. Vitamin D status as related to race and feeding type in preterm infants. Breastfeed Med. 2006;1(3):156-63. doi: 10.1089/bfm.2006.1.156. [PubMed: 17661592].

37. Boskabadi H, Maamouri G, Saghafi N, Nakhaei MHA. A New Approach to Prevent Neonatal Respiratory Distress Syndrome: A randomized clinical trial of $25(\mathrm{OH})$ D Injections before Preterm Delivery. Research Square. 2021. doi: 10.21203/rs.3.rs-570011/v1. 